\title{
3D-PRINTED EXPERIMENTAL DEVICE FOR THE PRODUCTION OF BIODEGRADABLE FIBROUS MATERIALS FOR BIOMEDICAL SCAFFOLD PURPOSES VIA THE WET ELECTROSPINNING METHOD
}

\author{
Jan KOVACICIN, Sarka HAUZEROVA, Lubos BEHALEK, David LUKAS, \\ Eva KUZELOVA KOSTAKOVA
}

Technical University of Liberec, Liberec, Czech Republic, EU, Jan.Kovacicin@tul.cz

https://doi.org/10.37904/nanocon.2021.4356

\begin{abstract}
The wet electrospinning method involves the use of a liquid instead of a solid or other type of collector for the collection of fabricated materials. This paper provides a description of an experimental laboratory wet electrospinning device, most of the various components of which were 3D printed by means of Fused Filament Fabrication (FFF) technology. The various morphologies of the biodegradable electrospun materials produced via the wet electrospinning technique are compared with those obtained via the classic needle electrospinning method employing SEM image analysis. The paper also provides a comparison with the structures and in-vitro viability of samples produced using the classic electrospinning approach and the wet electrospinning method with drying under both laboratory and freeze-drying conditions. The wet electrospinning freeze-drying method provides for the production of both high porosity and bulky materials. The in-vitro cell viability tests demonstrated that the wet electrospinning samples dried via the freeze-drying method evinced the highest rate of fibroblast proliferation. The novelty of the approach lies in its simplicity, the relatively low costs involved and the ease of the replication of the device for laboratory and low-quantity production purposes. Moreover, the various experiments conducted served both to prove the usefulness of the device and to determine that the resulting structure is suitable for cell growth, thus enabling the production of candidate materials for tissue engineering scaffolds.
\end{abstract}

Keywords: Wet electrospinning, scaffold, tissue engineering, 3D printer, cell viability

\section{INTRODUCTION}

The aim of the study was to compare 2D- and 3D-electrospun materials as candidate materials for use in tissue engineering applications and to evaluate their cell proliferation potential. Wet electrospinning (WET ES) technology represents a promising approach in terms of the production of materials suitable for tissue engineering purposes in the 3D form with larger pores and more voluminous structures than those produced via the classic method [6,7]. While the use of a classic solid electrospinning collector produces flat (2D) nanofiber layers that do not allow enough space for cells, and that are unable to replace the volume of larger tissues, WET ES produces a 3D structure with a higher degree of porosity that enables the proliferation and migration of cells, the exchange of nutrients and the waste disposal.

The wide-ranging experimental research led to the design and fabrication of a device comprising mainly components that were printed using a low cost 3D FFF printer [1], which allowed for the accurate replication, and the potential for the rapid and universal adaptation [2], of the device. Moreover, it was also designed to be user friendly and ready for use in a short time, including a simple wire connection system. The research of the base parameters for the experiments was inspired by a literature search [3-7] followed by the conducting of experiments aimed at determining the most suitable candidate materials. Once the final four materials had 
been selected, samples were produced for biological testing purposes and prepared for the cell viability testing phase. CCK8 cell viability tests were performed supplemented by scanning electron microscopy (SEM) and fluorescent microcopy analyses that enhanced the investigation of cell behavior within the material.

\section{MATERIALS AND METHOD}

The project consisted of four phases, the first of which comprised the design and construction of the WET ES experimental device, the second the determination of the candidate materials, followed by the fabrication of samples and, finally, the compilation of the biological testing plan. Following the testing of several materials, polymeric biocompatible and biodegradable PCL (polycaprolactone) was selected for experimentation due to its proven suitability for use in medical applications and its broad spinnability range $[3-5,10,11]$. The PCL was purchased from Merck (Germany) with two molecular weights (average Mn of 45,000 and Mn 80,000). Chloroform, ethanol, acetic acid, formic acid and acetone purchased from Penta (CZ) were used as the solvent systems (see Table 1); ethanol was also used as the liquid collector.

Table 1 The PCL solutions (designated G1 to G4) selected for biological testing. The first three differed in terms of the solvent, concentration and molecular mass Mn; G4 was a modified version of G3 with an increased polymer concentration. The average fiber diameters and standard deviations were calculated for each electrospun sample.

\begin{tabular}{|c|c|c|c|c|}
\hline Sample designation & G1 & G2 & G3 & G4 \\
\hline Material & PCL & PCL & PCL & PCL \\
\hline Concentration (w/w \%) & 20 & 10 & 12 & 15 \\
\hline Solvent & Chloroform & Chloroform/ethanol & $\begin{array}{l}\text { formic acid/acetic } \\
\text { acid/acetone }\end{array}$ & $\begin{array}{c}\text { formic acid/acetic } \\
\text { acid/acetone }\end{array}$ \\
\hline Solvent ratio $(w / w)$ & - & $8: 2$ & $1: 1: 1$ & $1: 1: 1$ \\
\hline $\begin{array}{l}\text { Average molecular mass } \\
{\left[\mathrm{Mn} * 10^{3}\right]}\end{array}$ & 45 & 80 & $45 / 80(w / w 1: 3)$ & $45 / 80(w / w 1: 3)$ \\
\hline Average fiber diameter [nm] & 6864 & $\begin{array}{c}9722 \\
625 \\
\text { *(bimodal values) } \\
\end{array}$ & 481 & 662 \\
\hline $\begin{array}{l}\text { Standard deviation of the fiber } \\
\text { diameter [nm] }\end{array}$ & 479 & $\begin{array}{c}5185 \\
129 \\
\text { *(bimodal values) }\end{array}$ & 241 & 142 \\
\hline
\end{tabular}

\subsection{Device design and fabrication}

In order to simplify the construction of the device, most of the components were fabricated, replicated and modified via 3D printing [2] using a Prusa MK3S 3D printer [1]. The use of conductive materials was minimized so as to ensure the maximum level of electrical safety in the vicinity of the High Voltage (HV) supply and to limit any potential electric field distortion. The device was constructed so as to facilitate simple one-piece transportation, rapid electric connection and the broadest possible range of setup parameters. The universal grip [8] allowed for the use of a wide range of spinning needles and other spinning electrodes (tips). The device included the relevant cable and tube arrangements, a non-conductive 3D printed lab platform jack [9] and a liquid bath dish, which was printed in black with rounded sides; the black color allowed for the enhanced observation of the lighter colored fiber structures in the dish and the rounded sides helped to minimize charge cumulation. The stainless electrode positioned in the middle enabled the connection of the ground potential from below the dish to the dish volume. Images of the device are shown in Figures $\mathbf{1}$ and $\mathbf{2}$. 

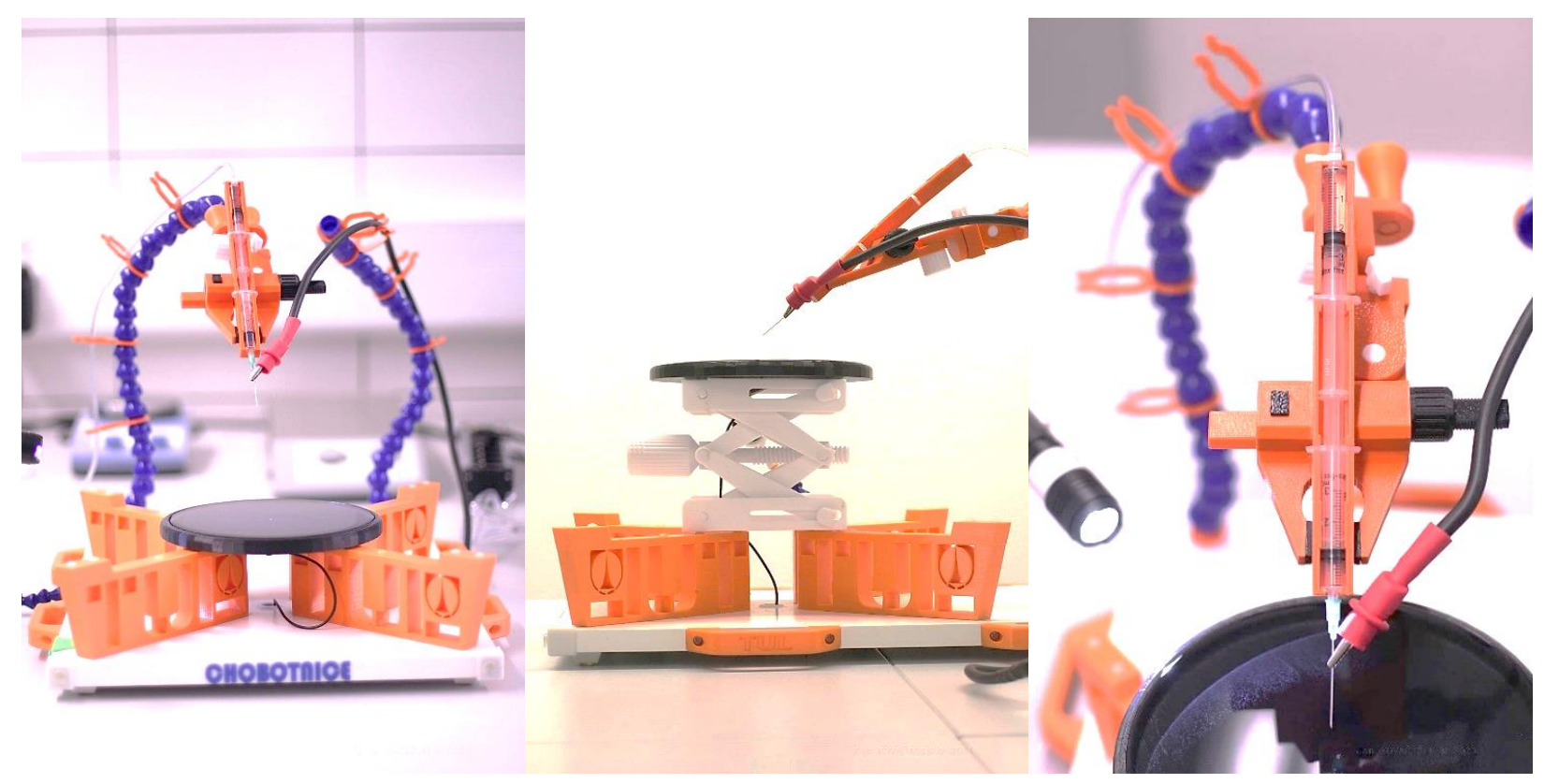

Figure 1 Images of the experimental wet electrospinning device. The image on the left shows the whole of the device, the middle image shows a side view of the platform with the polymeric 3D-printed jack [9] and the bath dish, and the image on the right shows the universal grip [8] and the hydraulic pressure syringe transmitter with a needle spinning tip.

\subsection{Biological testing}

Batch samples G1-G4 were subjected to cell viability testing using a $10 \%$ solution of CCK-8 counting test assay with 3 T3 mouse embryonic fibroblasts (MEFs). Four samples were tested on each testing day (days 1 , 3 and 7) with one negative control (NC) without seeded cells. The samples were transferred to fresh sterile microtitration plates prior to the CCK-8 testing procedure so as to ensure that only the cells in the samples were counted. Since the cell viability test compared the metabolic activity of the cells in the samples, it served for the evaluation of the suitability of the samples for cell proliferation purposes.

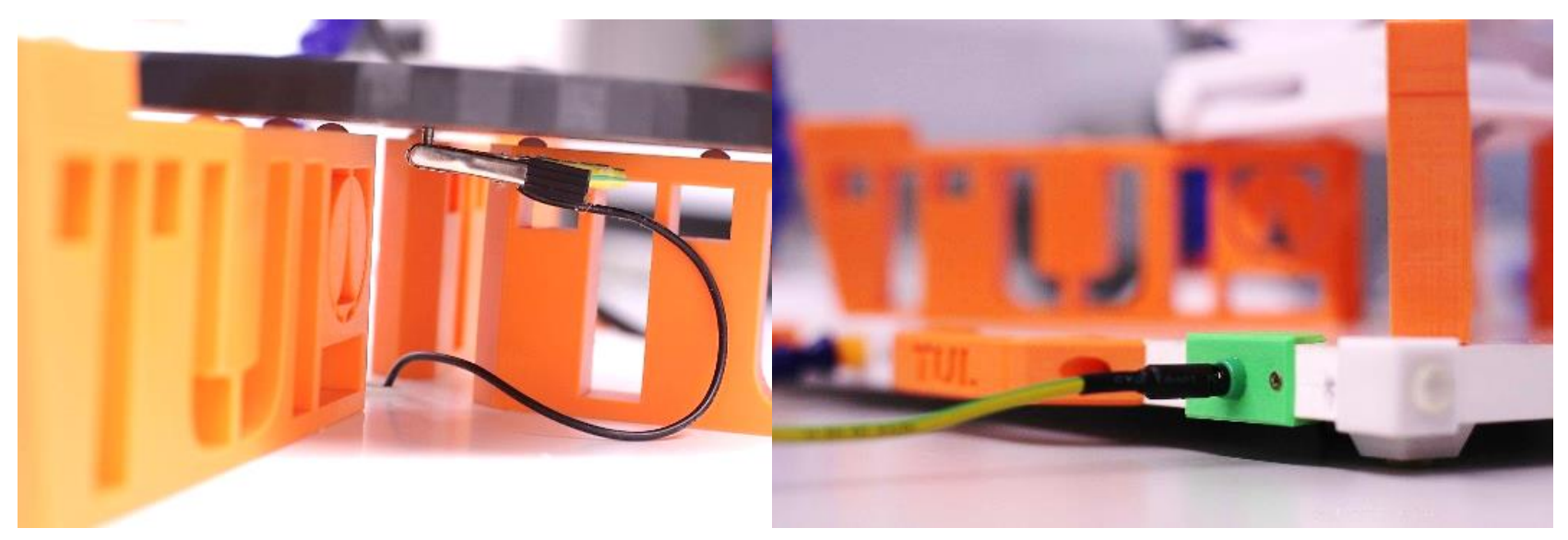

Figure 2 Further images of the experimental wet electrospinning device. The image on the left shows the platform, the grounding access point and the connection of the ground potential to the bath dish. The image on the right shows the ground input connector of the platform. 


\section{RESULTS}

The next stage involved the testing of the PCL with the selected solvents, supplemented by the evaluation of the spinnability and the submersion quality and the SEM image analysis. Using a scoring system and the SEM image evaluation, the best-performing candidate materials were selected for the production of the final samples (see Table 1 and Figure 3). The criteria for the selection of the samples for the biological testing phase comprised optimum spinnability performance, wettability by the collector liquid during WET ES and the variability of the structure (varying fiber diameters, the bimodal distribution of the fiber diameters in the sample, the smoothness or curliness of the fibers in the sample, etc.).

Batches of 15 samples were produced for each material (G1-G4). Batches were prepared of the WET samples along with batches of samples produced on a hard-flat metal collector. The WET samples were produced in an ethanol bath; the ethanol was replaced with water for lyophilization (freeze drying) purposes. With concern to the G4 material, a 3rd batch of 15 samples was produced in addition to the initial two batches; these samples were fabricated via the WET ES method but the ethanol base was allowed to evaporate naturally at room temperature in a fume hood. The batches of the WET lyophilized samples were observed to be both bulky and 3D symmetrical; the ethanol-evaporated WET ES samples were also seen to be 3D symmetrical but more compact than their lyophilized counterparts. The classic electrospinning samples were observed to be very flat. A comparison of the shapes and sizes of the samples is shown in Figure 4. All the samples were sterilized using ethylene oxide, which was allowed to evaporate for fourteen days.

Table 2 List of the mean absorbances and calculated growth rates. The cell metabolic activity as represented by the absorbance indicates that the planar samples exhibited the best initial seeding performance, whereas the WET 3D samples evinced the best growth rates.

\begin{tabular}{|l|c|c|c|c|c|}
\cline { 2 - 6 } \multicolumn{1}{c|}{} & Absorbance & Absorbance & Growth rate & Absorbance & Growth rate \\
\cline { 2 - 6 } \multicolumn{1}{c|}{} & Day 1 & Day 3 & Days 1 to 3 & Day 7 & Days 3 to 7 \\
\hline PC & 0.193263 & 1.186075 & $\mathbf{5 1 4} \%$ & 1.743300 & $\mathbf{4 7} \%$ \\
\hline G1 ES & 0.007838 & 0.024300 & $\mathbf{2 1 0} \%$ & 0.154538 & $\mathbf{5 3 6 \%}$ \\
\hline G2 ES & 0.010000 & 0.032375 & $\mathbf{2 2 4} \%$ & 0.304763 & $\mathbf{8 4 1} \%$ \\
\hline G4 ES & 0.037438 & 0.101538 & $\mathbf{1 7 1} \%$ & 0.393975 & $\mathbf{2 8 8} \%$ \\
\hline G1 WET ES & 0.032250 & 0.103388 & $\mathbf{2 2 1} \%$ & 0.539800 & $\mathbf{4 2 2} \%$ \\
\hline G2 WET ES & 0.005050 & 0.004100 & $\mathbf{- 1 9 \%}$ & 0.080850 & $\mathbf{1 8 7 2} \%$ \\
\hline G3 WET ES & 0.000010 & 0.000375 & $\mathbf{3 6 5 0} \%$ & 0.081200 & $\mathbf{2 1 5 5 3 \%}$ \\
\hline G4 WET ES & 0.000325 & 0.007425 & $\mathbf{2 1 8 5} \%$ & 0.182825 & $\mathbf{2 3 6 2} \%$ \\
\hline G4 WET ES MOD & 0.006125 & 0.008450 & $\mathbf{3 8} \%$ & 0.096625 & $\mathbf{1 0 4 3} \%$ \\
\hline
\end{tabular}

The results of the biological testing revealed lower initial cell counts in the bulky samples fabricated using the WET ES method, Figure 5 and Table 2. The absorbance-cell activity values were observed to be higher for the planar samples produced using the classic ES method; this was most likely due to the initial seeding of a higher number of cells in structures with smaller pores and the fact that the samples lay flat upon the bottom of the cultivation well plates, thus allowing cells to migrate into the sample from the bottom. However, over time, the cells in the planar samples did not proliferate well due to the small size of the pores and/or a lack of space. Conversely, the WET samples initially seeded to a significantly lower extent; the initial cell count based on the absorbance was lower due to the presence of large pores which failed to physically "hook" the cells during the initial cell seeding period. However, over time, the WET ES samples evinced rapid cell proliferation with a very rapid growth rate, i.e. $21553 \%$ compared to a maximum growth rate of $422 \%$ recorded for the flat 
samples produced via the classic collector electrospinning method (Figure 6). The growth rate was calculated as an increase in the cell number represented by the absorbance, and was calculated between the 1st and 3rd days and the 3rd and 7th days. The explanation for the rapid growth rate of the WET bulky samples lies in their higher pore sizes and large 3D structures, which allowed the cells to proliferate and migrate within the bulky structures. The 3D structures contained more space for the cells and allowed for the exchange of nutrients and the disposal of waste. It is believed that the cell count was lower for the WET samples on the first day since the larger pores did not retain the cells in the samples, which resulted in their remaining on the bottom of the microtitration plates, i.e. the main reason comprised the large pores of the WET samples. On the other hand, the larger pores subsequently allowed the cells to proliferate and migrate freely into the volume of the samples; hence, the enormous growth rates recorded for the WET samples. It is recommended that further experiments should be conducted on 3D WET ES materials in the future that allow for cell viability testing over longer time periods of up to 14 days; moreover, other cell types should be considered and an efficient seeding technique developed specifically for bulky porous materials.

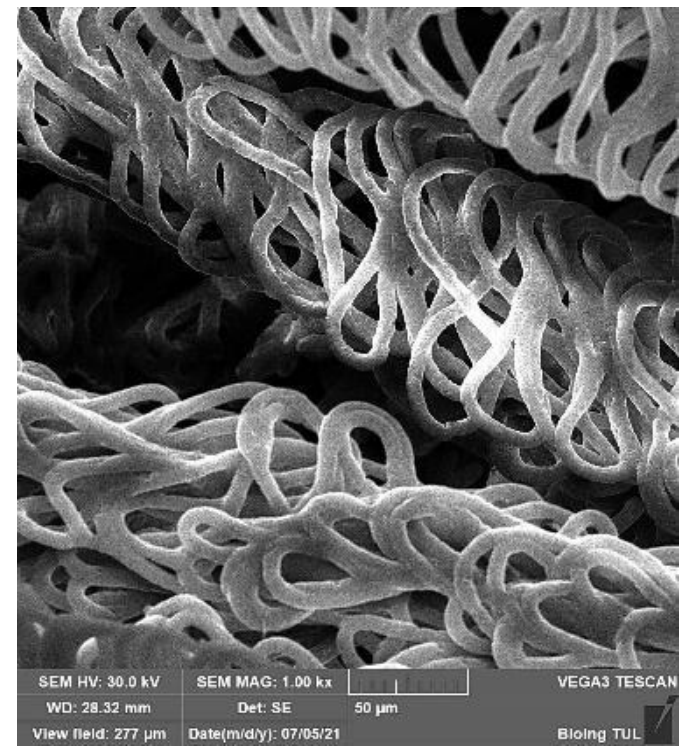

G1

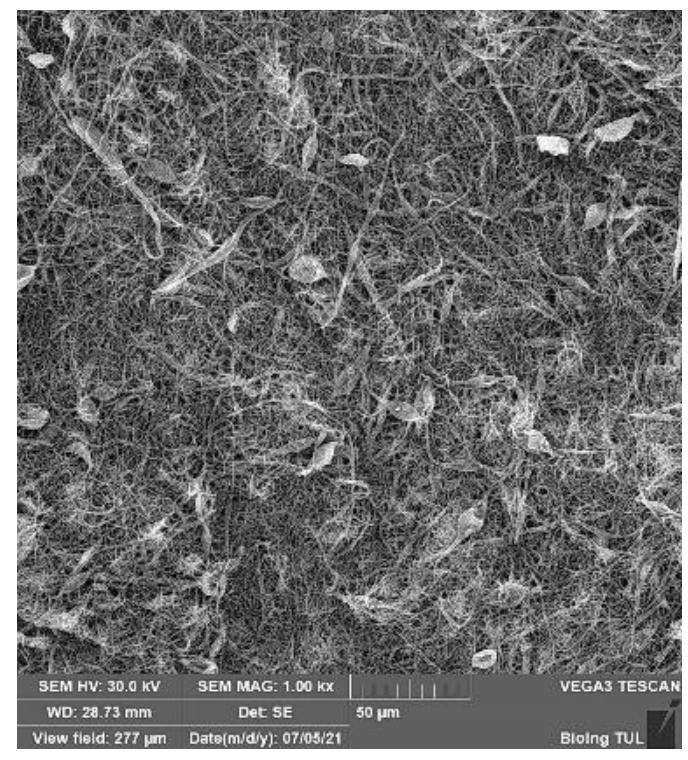

G3
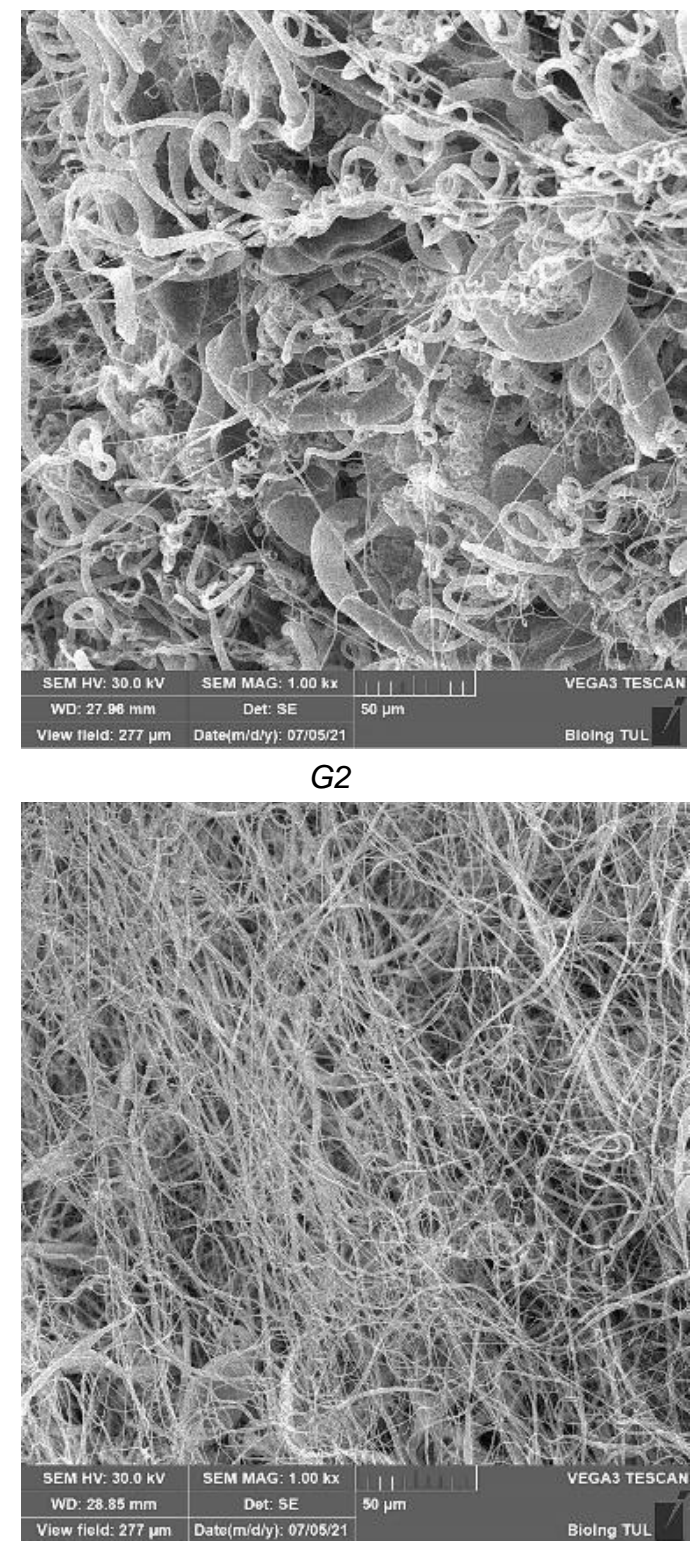

G4

Figure 3 SEM images of the wet electrospun fibrous materials fabricated from polymeric solutions G1 - G4. The scale bar represents 50 micrometers. 


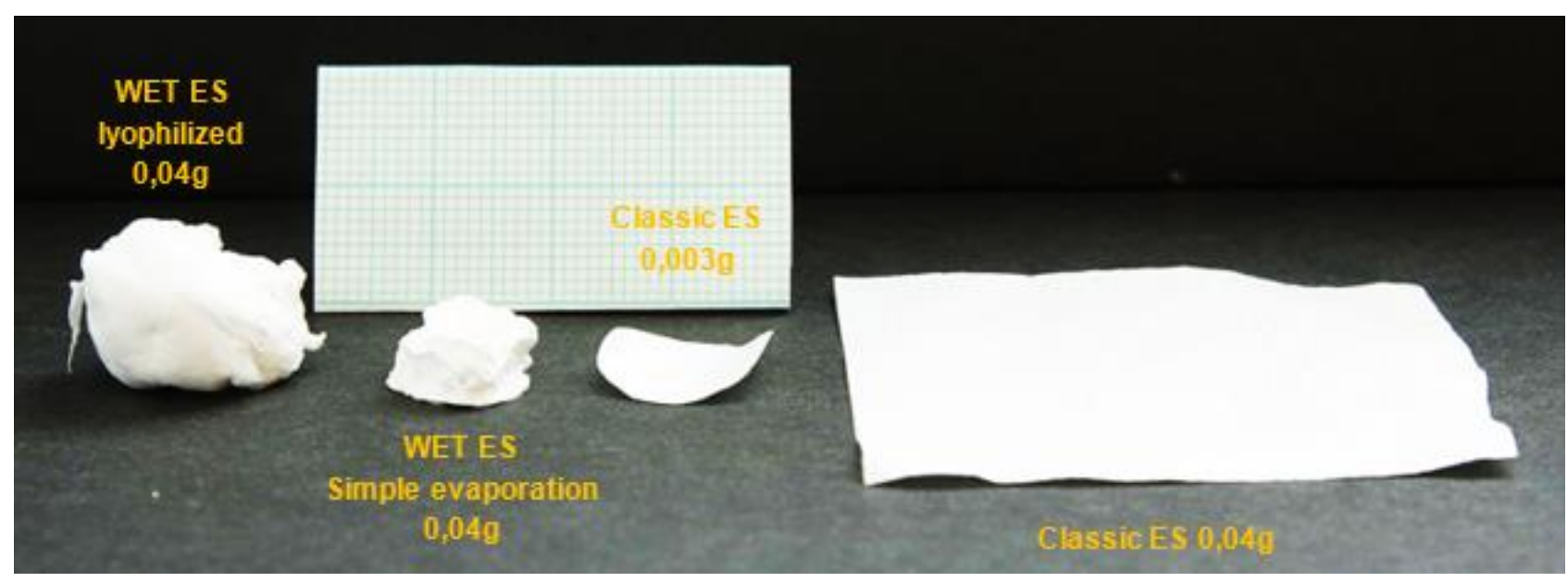

Figure 4 Images of the G4 material samples; from left to right: a WET electrospinning lyophilized bulky material, a WET ES material dried via ethanol evaporation, and a classic flat electrospinning sample trimmed

to the testing size. The image on the right is included so as to provide a comparison of a classic flat electrospinning sample with the same weight as the bulky WET lyophilized sample shown on the far left.

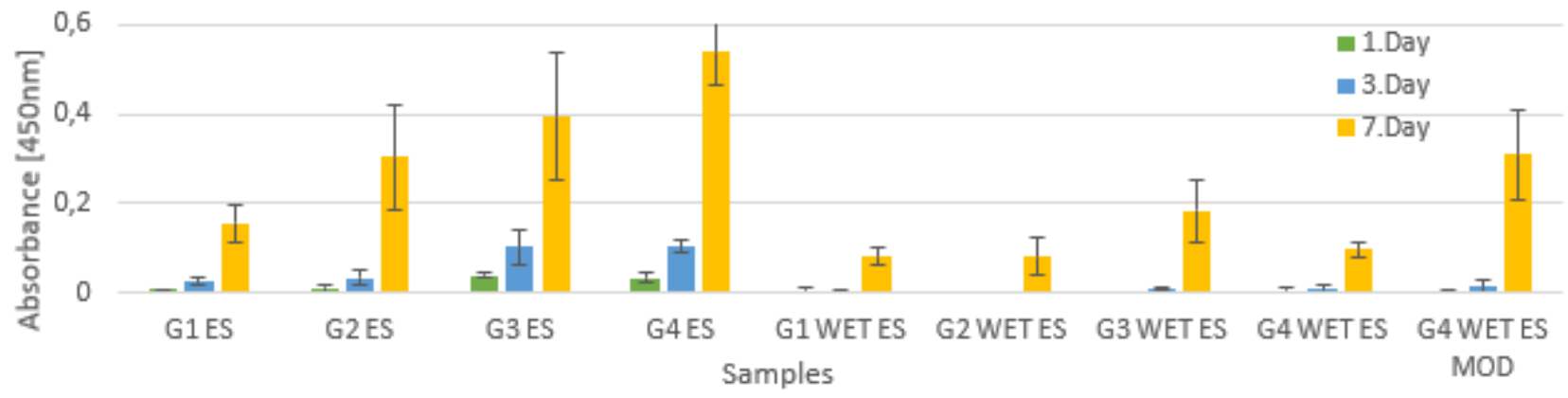

Figure 5 The cell count as represented by the spectrophotometry absorbance; the first four G1-4 ES samples were planar (flat) fabricated via the classic hard electrospinning technique; the next four G1-4 WET ES samples were fabricated via the WET electrospinning technique and dried by means of lyophilization; the final G4 WET ES MOD sample was dried via the natural evaporation of the ethanol.

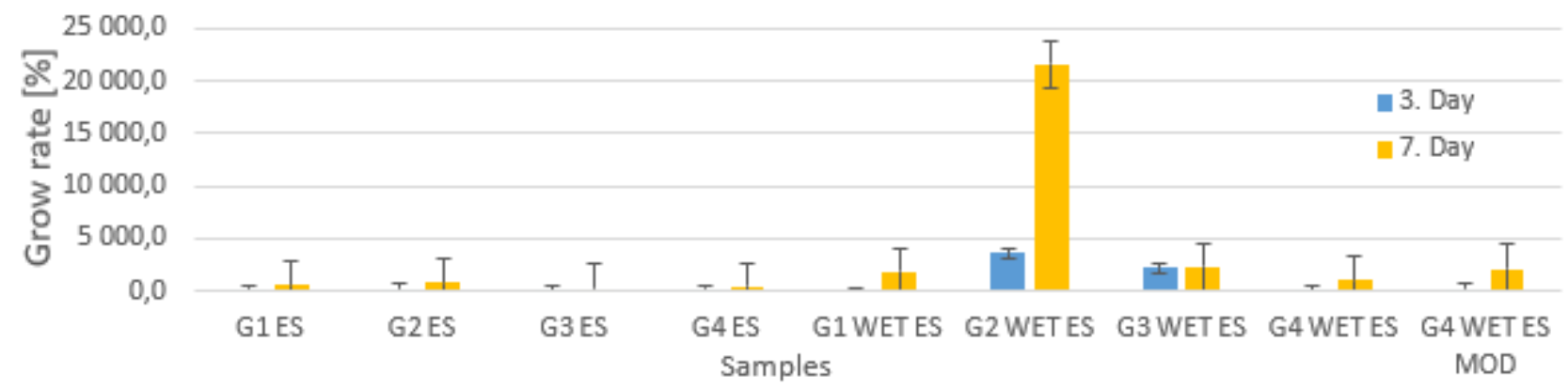

Figure 6 Growth rates representing the cell gain between the 1st and 3rd days (designated 3. Day) and the 3rd and 7th days (designated 7. Day); the first four G1-4 ES samples were planar (flat) fabricated via the classic hard collector electrospinning technique; the next four G1-4 WET ES samples were fabricated via the WET electrospinning technique and dried by means of lyophilization; the final G4 WET ES MOD sample was dried via the natural evaporation of the ethanol. 


\section{CONCLUSIONS}

The electrospinning of fibrous materials in a liquid collector leads to significant differences in terms of the final structure of the material compared to materials electrospun using conventional flat collectors. Moreover, the three-dimensional character of wet electrospun materials revealed interesting differences in terms of the initial in-vitro testing results; in particular, a significant difference in the cell proliferation rate. While initial seeding was observed to be more effective on the planar structures, the cell proliferation rate was much more pronounced for the 3D structures fabricated via the WET ES method (a $21553 \%$ increase from day 3 to day 7 for sample G2 WET ES) than for the best-performing planar sample (an $841 \%$ increase from day 3 to day 7). It is suggested that the vastly improved proliferation rate of the bulkier tissues indicates the true potential of the developed 3D materials for use in tissue engineering applications.

\section{ACKNOWLEDGEMENTS}

The authors express their gratitude for the financial support provided by the "Study of physical networks in polymer materials" grant project of the "PURE Technical University of Liberec Grant Program", Ref. PURE-2020-4007.

\section{REFERENCES}

[1] Original Prusa i3 MK3S+ - Prusa Research a.s.. - 3D print a 3D printers by Josef Průša [Online].

[2] SELLS, E., BAILARD, S., SMITH, Z., BOWYER, A., OLLIVER, V. RepRap: The Replicating Rapid Prototyper: Maximizing Customizability by Breeding the Means of Production. In: Handbook of Research in Mass Customization and Personalization [Online]. World Scientific Publishing Company. 2009, pp. 568-80.

[3] WOODRUFF, M.A., HUTMACHER, D.W. The return of a forgotten polymer-Polycaprolactone in the 21st century. Prog Polym Sci. Vol. 35, no. 10, pp.1217-56.

[4] KIM, M.S., KIM, G.H. Highly porous electrospun 3D polycaprolactone/ $\beta$-TCP biocomposites for tissue regeneration. Mater Lett. Vol. 120, pp. 246-50.

[5] KOSTAKOVA, E., SEPS, M., POKORNY, P., LUKAS, D. Study of polycaprolactone wet electrospinning process. Express Polym Lett. 2014; vol. 8, no. 8, pp. 554-64.

[6] POKORNY, P., KOSTAKOVA, E., SANETRNIK, F., MIKES, P., CHVOJKA, J., KALOUS, T., et al. Effective AC needleless and collectorless electrospinning for yarn production. Phys Chem Chem Phys. 2014, vol. 16, no. 48, pp. 26816-22.

[7] YOKOYAMA Y, HATTORI S, YOSHIKAWA C, YASUDA Y, KOYAMA H, TAKATO T, et al. Novel wet electrospinning system for fabrication of spongiform nanofiber 3-dimensional fabric. Mater Lett. 2009; vol. 63, no. 9-10, pp. 754-6.

[8] MaH - Modular Arm Helper - No support - Easy print by b03tz - Thingiverse [Online].

[9] Platform Jack [Fully Assembled, No Supports] by Intentional3D - Thingiverse [Online].

[10] DOMINGOS, M., INTRANUOVO, F., GLORIA, A., GRISTINA, R., AMBROSIO, L., BÁRTOLO, PJ., et al. Improved osteoblast cell affinity on plasma-modified 3-D extruded PCL scaffolds. Acta Biomater. 2013; vol. 9, no. 4, pp. 5997-6005.

[11] ZHANG, M., LIN, H., WANG, Y., YANG, G., ZHAO, H., SUN, D. Fabrication and durable antibacterial properties of 3D porous wet electrospun RCSC/PCL nanofibrous scaffold with silver nanoparticles. Appl Surf Sci. 2017, vol. 414 , pp. 52-62. 